\title{
Optimal Power Allocation for OFDM-Based Wire-Tap Channels with Arbitrarily Distributed Inputs
}

\author{
Haohao Qin`, Yin Sun`, Xiang Chen, Ming Zhao, Jing Wang \\ State Key Laboratory on Microwave and Digital Communications \\ Tsinghua National Laboratory for Information Science and Technology \\ Department of Electronic Engineering, Tsinghua University, Beijing 100084, \\ P. R. China \\ \{haohaoqin07, sunyin02, chenxiang98, zhao.ming29\}@gmail.com \\ wangj@mail.tsinghua.edu.cn
}

\begin{abstract}
In this paper, we investigate power allocation that maximizes the secrecy rate of orthogonal frequency division multiplexing (OFDM) systems under arbitrarily distributed inputs. Considering commonly assumed Gaussian inputs are unrealistic, we focus on secrecy systems with more practical discrete distributed inputs, such as PSK, QAM, etc. While the secrecy rate achieved by Gaussian distributed inputs is concave with respect to the transmit power, we have found and rigorously proved that the secrecy rate is non-concave under any discrete inputs. Hence, traditional convex optimization methods are not applicable any more. To address this non-concave power allocation problem, we propose an efficient algorithm. Its gap from optimality vanishes asymptotically at the rate of $O(1 / \sqrt{N})$, and its complexity grows in the order of $O(N)$, where $N$ is the number of sub-carriers. Numerical results are provided to illustrate the efficacy of the proposed algorithm.
\end{abstract}

Key words: OFDM wire-tap channel, arbitrarily distributed inputs, duality theory, non-convex problem, optimal power allocation

\section{Introduction}

In recent years, many privacy sensitive wireless services, such as pushmail, mobile wallet, Microblogging, etc, have become more and more popular. But, due to the broadcast nature of wireless channels, security problems and challenges are also accompanying with the growing up of those privacy services. The security of wireless communications is commonly supported by cryptographic techniques employed at upper layer. However, this traditional method faces several challenges, such as the emergence of new cracking algorithms and increasing computational capability of eavesdroppers. Recently, physical layer security, a method that can supplement upper layer security, has received considerable attentions [1.

\footnotetext{
* Haohao Qin and Yin Sun contribute equally to this work.
} 
Physical layer security was firstly studied from an information-theoretic perspective in 2, where the concept of "wire-tap channel" was introduced to illustrate the channel with three terminals, transmitter, legitimate receiver and eavesdroppers, and secrecy rate was defined as the maximum achievable data rate from the transmitter to its legitimate receiver while keeping the eavesdropper completely ignorant of the secret massage. Later, the research in this field was extended to various scenes, such as Gaussian wire-tap channel [3- 4], multiple input multiple output (MIMO) channel [5]-[7], orthogonal frequency division multiplexing (OFDM) channel []-[1], etc.

Recently, OFDM-based secure communications have obtained much attention for its capability of countermining the dispersive of wideband wireless channels and enhance secrecy rate [8-[11]. Optimal power allocation of OFDM-based wire-tap channels is investigated in [8]-[10] under Gaussian inputs. In practical system, due to Gaussian inputs' infinite peak-to-average ratio, finite discrete constellation:2, such as PSK, QAM (see Fig 1](a)), are used instead. In this paper, we investigate optimal power allocation for OFDM-based wire-tap channels under arbitrarily distributed channel inputs.

We have found and rigorously proved that the secrecy rate is non-concave under any discrete constellations, while the secrecy rate achieved by Gaussian distributed channel inputs was found to be concave with respect to the transmit power [8]-[10]. Therefore, the optimal power allocation strategy for OFDM-based wire-tap channels with Gaussian inputs [8]-[10] is not applicable any more to the considered problem. To address this non-concave power allocation problem, we propose an efficient power allocation algorithm. Its gap from optimality vanishes asymptotically at the rate of $O(1 / \sqrt{N})$, and its complexity grows in the order of $O(N)$, where $N$ is the number of sub-carriers. Numerical results are provided to illustrate the efficiency of the proposed algorithm.

The remainder of this paper is organized as follows. Section 2 presents the system model and power allocation problem is formulated. Optimal power allocation for arbitrarily distributed inputs is given in Section 3. Numerical results and conclusions are provided in Section 4 and Section 5, respectively.

\section{System Model and Problem Formulation}

Consider an OFDM-based wire-tap channel with a transmitter, a legitimate receiver and an eavesdropper, where the eavesdropper intends to extract the confidential message transmitted from transmitter to legitimate receiver (see Fig. [1 (b)). There are $N$ sub-carriers and the transmitter's signal in each sub-carrier follows an arbitrary but predetermined distribution, which can be either continuous constellations, such as Gaussian distribution, or finite discrete constellations, including PSK, QAM, etc (see Fig. 11(a)).

The transmitted signal over the $i$ th sub-carrier is denoted as $x_{i}$, described as

\footnotetext{
2 The words "distribution" and "constellation" are used alternatively throughout the paper.
} 


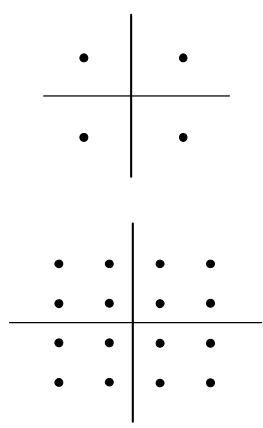

(a)

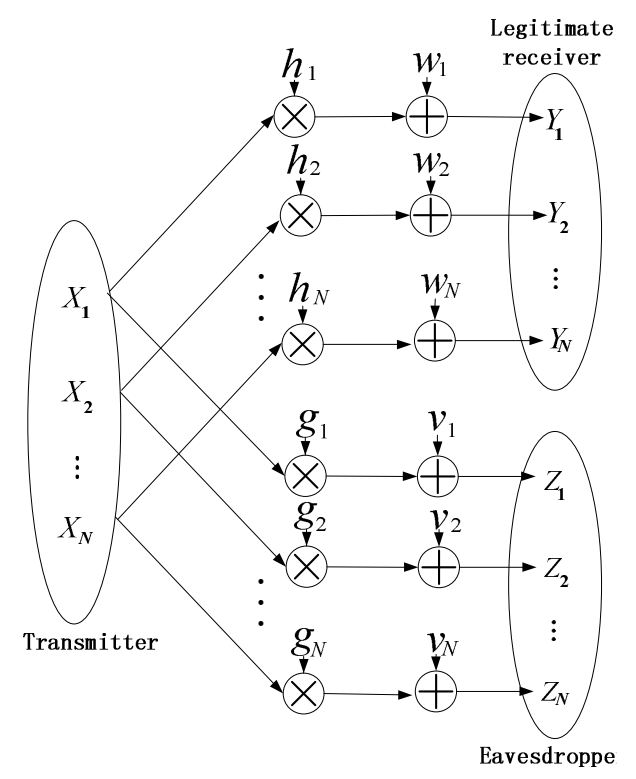

(b)

Fig. 1. (a). Finite discrete constellations: QPSK and 16QAM, (b). OFDM-based wiretap channel

$$
x_{i}=\sqrt{p_{i}} s_{i}, i=1, \ldots, N \text {, }
$$

where $p_{i}$ is the power ratio between transmission signal $x_{i}$ and the noise, and $s_{i}$ represents the normalized channel inputs with predetermined distribution. Then power constraint can be readily shown to be

$$
\frac{1}{N} \sum_{i=1}^{N} p_{i} \leq P
$$

where $P$ is total available transmit power.

The received signals at the legitimate receiver and eavesdropper are given by

$$
\begin{array}{ll}
y_{i}=h_{i} \sqrt{p_{i}} s_{i}+w_{i}, & i=1, \cdots, N, \\
z_{i}=g_{i} \sqrt{p_{i}} s_{i}+v_{i}, & i=1, \cdots, N,
\end{array}
$$

respectively, where the $w_{i}$ and $v_{i}$ are zero-mean complex Gaussian noises with unit variance; $h_{i}$ and $g_{i}$ are the complex channel coefficients of $i$ th sub-carrier. According to the information theoretical studies of [8], the secrecy rate from transmitter to its legitimate receiver is

$$
\sum_{i=1}^{N}\left[I\left(s_{i} ; h_{i} \sqrt{p_{i}} s_{i}+w_{i}\right)-I\left(s_{i} ; g_{i} \sqrt{p_{i}} s_{i}+v_{i}\right)\right]^{+},
$$


where $[x]^{+} \triangleq \max \{x, 0\}$, and $I(x ; y)$ denotes the mutual information between random variables $x$ and $y$. The expression in (5) is quite illuminating: the secrecy rate of each sub-channel is non-negetive; if it is positive, it is exactly the data rate difference of the legitimate and eavesdropping channels; the total secrecy rate is simply the sum secrecy rate of all the $N$ sub-carriers.

For fixed constellations of $\left\{s_{i}\right\}_{i=1}^{N}$, we need to optimize the power allocation to obtain the maximal secrecy rate. The optimization problem can be cast as follows,

$$
\begin{aligned}
R^{*}=\max _{\mathbf{p}} & R_{s}(\mathbf{p}) \triangleq \frac{1}{N} \sum_{i=1}^{N}\left[I\left(s_{i} ; h_{i} \sqrt{p_{i}} s_{i}+w_{i}\right)-I\left(s_{i} ; g_{i} \sqrt{p_{i}} s_{i}+v_{i}\right)\right]^{+} \\
\text {s.t. } & \frac{1}{N} \sum_{i=1}^{N} p_{i} \leq P, \\
& \mathbf{p} \geq 0
\end{aligned}
$$

where $\mathbf{p} \in \mathcal{R}^{N}$ is transmit power vector for $N$ sub-carriers, i.e., $\mathbf{p}=\left\{p_{1}, p_{2}, \ldots, p_{N}\right\}$, and $R^{*}$ denotes the optimal value. For the facility of the following analysis, let $R_{s, i}\left(p_{i}\right)$ denote the ingredients of $R_{s}(\mathbf{p})$, i.e.,

$$
R_{s, i}\left(p_{i}\right) \triangleq\left[I\left(s_{i} ; h_{i} \sqrt{p_{i}} s_{i}+w_{i}\right)-I\left(s_{i} ; g_{i} \sqrt{p_{i}} s_{i}+v_{i}\right)\right]^{+} .
$$

\section{Optimal Power Allocation under Arbitrarily Distributed Channel Inputs}

\subsection{Non-concavity of the Secrecy Rate $R_{s}(p)$}

If $s_{i}$ follows Gaussian distribution, the secrecy rate $R_{s}(\mathbf{p})$ in (6) has explicit expression [4, i.e.,

$$
R_{s}^{G}(\mathbf{p})=\frac{1}{N} \sum_{i=1}^{N}\left[\log _{2}\left(1+\left|h_{i}\right|^{2} p_{i}\right)-\log _{2}\left(1+\left|g_{i}\right|^{2} p_{i}\right)\right]^{+} .
$$

It can be checked that $R_{s}^{G}(\mathbf{p})$ is a concave function of $\mathbf{p}$. Hence problem (6) is a convex optimization problem. The ingredients of $R_{s}^{G}(\mathbf{p})$ are illustrated in the left part of Fig. 2. One can observe that $\log _{2}\left(1+\left|h_{i}\right|^{2} p_{i}\right), \log _{2}\left(1+\left|g_{i}\right|^{2} p_{i}\right)$ and $\log _{2}\left(1+\left|h_{i}\right|^{2} p_{i}\right)-\log _{2}\left(1+\left|g_{i}\right|^{2} p_{i}\right)$ are all concave, provided that $\left|h_{i}\right|^{2}>\left|g_{i}\right|^{2}$. In [8]-[10], utilizing the convexity structure of problem (6), authors obtained the optimal power allocation shown as follows,

$$
p_{i}^{*}=\left\{\begin{array}{cl}
\frac{-\left(\left|h_{i}\right|^{2}+\left|g_{i}\right|^{2}\right)+\sqrt{\left(\left|h_{i}\right|^{2}+\left|g_{i}\right|^{2}\right)^{2}-4\left|h_{i}\right|^{2}\left|g_{i}\right|^{2} \frac{u+\left|g_{i}\right|^{2}-\left|h_{i}\right|^{2}}{u}}}{2\left|h_{i}\right|^{2}\left|g_{i}\right|^{2}} & \text {,if }\left|h_{i}\right|^{2}-\left|g_{i}\right|^{2}>u \\
0 & \text {, others, }
\end{array}\right.
$$

where the Lagrange multiplier $u$ is chosen to meet the power constraint, 


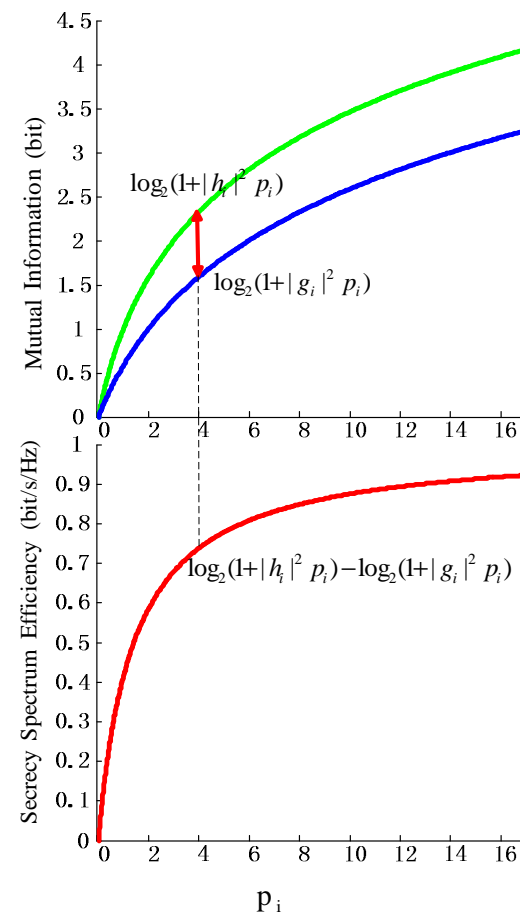

(a)

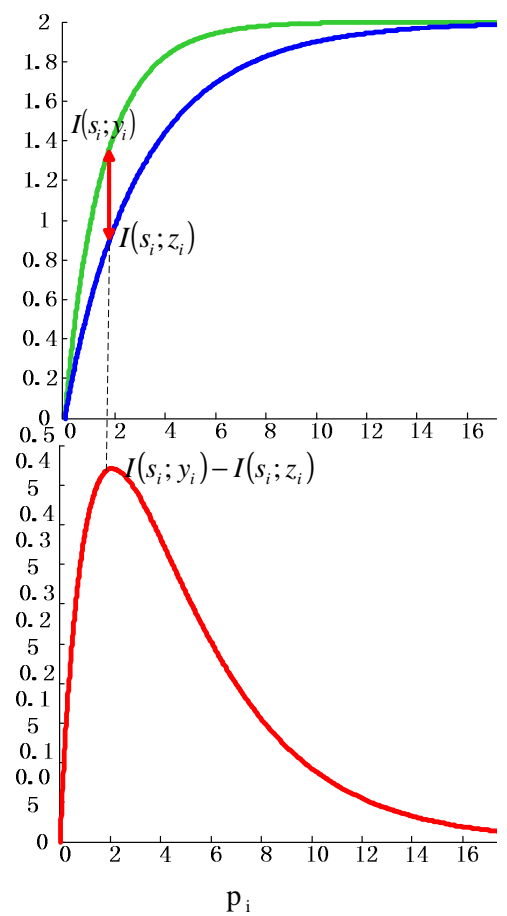

(b)

Fig. 2. (a). Secrecy rate achieved by Gaussian distributed inputs. (b). Secrecy rate achieved by discrete inputs (eg. QPSK). Here $h_{i} \geq g_{i}$.

$$
\frac{1}{N} \sum_{i=1}^{N} p_{i}=P
$$

One may expect that the concavity of $R_{s}(\mathbf{p})$ still holds under general input distributions. Unfortunately, our investigation shows that this is not true, which is formally presented in the following proposition.

Proposition 1. The secrecy rate function $R_{s}(\boldsymbol{p})$ under any finite discrete constellations is non-concave with respect to $\boldsymbol{p}$.

Proof. When $p_{i}=0$, one can derive $I\left(s_{i} ; y_{i}\right)=I\left(s_{i} ; z_{i}\right)=0$; when $p_{i}=+\infty$, we have $I\left(s_{i} ; y_{i}\right)=I\left(s_{i} ; z_{i}\right)=H\left(s_{i}\right)$, where $H(x)$ is entropy of $x$. Therefore, $R_{s, i}(0)=R_{s, i}(+\infty)=0$.

According to [15,

$$
\frac{\partial I(s ; \sqrt{p} s+n)}{\partial p}=\operatorname{MMSE}(p)
$$

$\operatorname{MMSE}(p)$ is defined as

$$
\operatorname{MMSE}(p) \triangleq \mathbb{E}\left[|s-\mathbb{E}(s \mid \sqrt{p} s+n)|^{2}\right]
$$



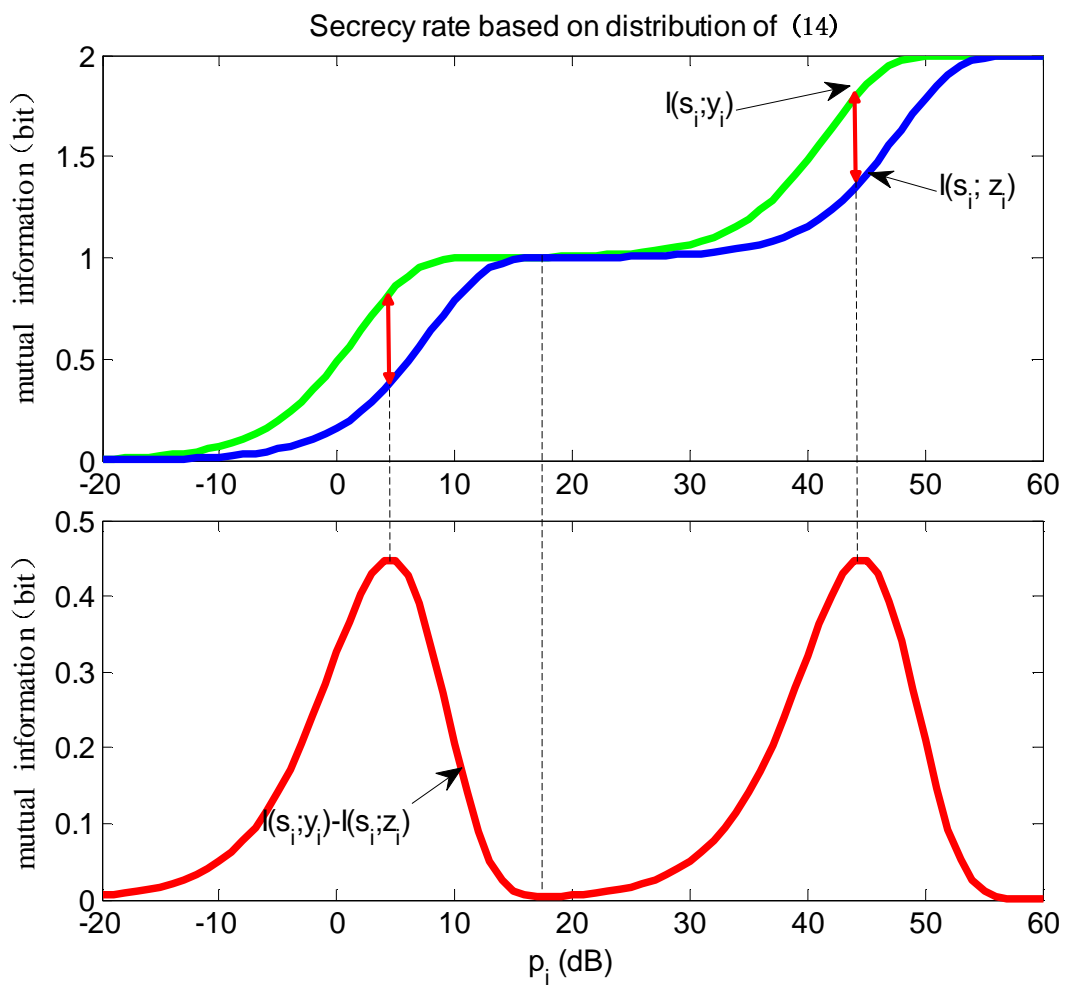

Fig. 3. Secrecy rate achieved by distribution of (14).

where $\mathbb{E}[x]$ is the expectation of random variable $x ; \mathbb{E}[x \mid y]$ is the conditional expectation of $x$ for given $y$. By (11), the derivative of $R_{s, i}\left(p_{i}\right)$ at $p_{i}=0$ is given by ${ }^{3}$

$$
\left.R_{s, i}^{\prime}\left(p_{i}\right)\right|_{p_{i}=0}=\left[\left|h_{i}\right|^{2}-\left|g_{i}\right|^{2}\right]^{+}>0
$$

which indicates that there must exist a $\hat{p}_{i}>0$ that $R_{s, i}\left(\hat{p}_{i}\right)>0$. According to the Lagrange's mean value theorem [17, it must have a point $\tilde{p}_{i} \in\left[\hat{p}_{i},+\infty\right]$ with negative slop $R_{s, i}^{\prime}\left(\tilde{p}_{i}\right)<0$.

Assume $R_{s, i}\left(p_{i}\right)$ is concave, then the inequality $R_{s, i}\left(p_{i}\right) \leq R_{s, i}\left(\tilde{p}_{i}\right)+R_{s, i}^{\prime}\left(\tilde{p}_{i}\right)\left(p_{i}-\right.$ $\tilde{p}_{i}$ ) holds [18, which indicates $R_{s, i}(+\infty)=-\infty$. This contradicts $R_{s, i}(+\infty)=0$. Therefore, the concavity assumption is not true, and Proposition 1 holds.

Two evidentiary examples are provided to illustrate Proposition 1.

The first example is QPSK. The curves of $I\left(s_{i} ; y_{i}\right), I\left(s_{i} ; z_{i}\right)$ and $R_{s, i}\left(p_{i}\right)$ versus $p_{i}$ are shown in right part of Fig. 2, and they are in accordance with the statements in the proof of Proposition 1.

The second example considers a 4 points PAM constellation with non-uniform spacing. The probability mass function of $s_{i}$ is given by

\footnotetext{
${ }^{3}$ Only the sub-carriers that satisfy $\left|h_{i}\right|^{2}>\left|g_{i}\right|^{2}$ are considered, as $R_{s, i}\left(p_{i}\right) \equiv 0$ for those sub-carriers with $\left|h_{i}\right|^{2} \leq\left|g_{i}\right|^{2}$, which do not affect the concavity of $R_{s}(\mathbf{p})$.
} 


$$
P_{s_{i}} \sim\left[\begin{array}{cccc}
-51 L & -50 L & 50 L & 51 L \\
0.25 & 0.25 & 0.25 & 0.25
\end{array}\right]
$$

where $L$ is a normalization parameter to maintain unit variance. Figure 3 shows the secrecy rate $R_{s, i}\left(p_{i}\right)$ for this case. It is interesting that the $R_{s, i}\left(p_{i}\right)$ has two peaks. Hence, it is definitely non-concave. We note that the mutual information $I\left(s_{i} ; y_{i}\right)$ and $I\left(s_{i} ; z_{i}\right)$ are concave with respect to $p_{i}$ in linear scale [16].

\subsection{Optimal Power Allocation Solution of Problem (6)}

Although problem (6) is non-convex, there are still some efficient algorithms to solve it and obtain near-optimal solutions. One of them is the Lagrangian duality method [18]. Some recent studies [12]-[14] showed that, for certain non-convex structures, asymptotic optimal performance can be achieved by this method.

The Lagrangian of problem (6) is given by

$L(\mathbf{p}, u)=\frac{1}{N} \sum_{i=1}^{N}\left[I\left(s_{i} ; h_{i} \sqrt{p_{i}} s_{i}+w_{i}\right)-I\left(s_{i} ; g_{i} \sqrt{p_{i}} s_{i}+v_{i}\right)\right]^{+}+u\left(P-\frac{1}{N} \sum_{i=1}^{N} p_{i}\right)$,

where $u$ is Lagrangian dual variable. The corresponding dual function can then be written as

$$
g(u) \triangleq \max _{\mathbf{p} \geq 0} L(\mathbf{p}, u)
$$

Hence the dual problem formulation of problem (6) can be readily expressed as

$$
D^{*}=\min _{u \geq 0} g(u)
$$

where $D^{*}$ denotes the optimal dual value. Since the objective function of primal problem (6) is non-concave, there is a positive gap between $R^{*}$ and $D^{*}$, i.e., $D^{*}-R^{*}>0$. However, according to the recent studies of Luo and Zhang [12], [13, asymptotic strong duality holds for problem (6), i.e. the duality gap $D^{*}-R^{*}$ goes to zero as $N \rightarrow \infty$, as is expressed in the following proposition:

Proposition 2. If the channel coefficients $g_{i}$ and $h_{i}$ are Lipschitz continuous and bounded in the sense

$$
\begin{aligned}
& || h_{i}|-| h_{j}|| \leq L_{h} \frac{|i-j|}{N}, \forall i, j \in\{1,2, \ldots, N\} \\
& || g_{i}|-| g_{j}|| \leq L_{g} \frac{|i-j|}{N}, \forall i, j \in\{1,2, \ldots, N\}
\end{aligned}
$$

where $L_{h}, L_{g}>0$ is the Lipschitz constant. Then we have

$$
0 \leq D^{*}-R^{*} \leq O\left(\frac{1}{\sqrt{N}}\right)
$$


Proof. According to (11) and (12), we have 15]

$$
0 \leq \frac{\partial I(s ; \sqrt{p} s+n)}{\partial p}=\operatorname{MMSE}(p) \leq \mathbb{E}\left[|s|^{2}\right]=1,
$$

which implies that

$$
\begin{gathered}
\frac{\partial R_{s, i}(p)}{\partial p}=\left|h_{i}\right|^{2} \operatorname{MMSE}\left(\left|h_{i}\right|^{2} p\right)-\left|g_{i}\right|^{2} \operatorname{MMSE}\left(\left|g_{i}\right|^{2} p\right) \leq\left|h_{i}\right|^{2}, \\
\frac{\partial R_{s, i}(p)}{\partial\left|h_{i}\right|}=2 p\left|h_{i}\right| \operatorname{MMSE}\left(\left|h_{i}\right|^{2} p\right) \leq 2 p\left|h_{i}\right| \\
\frac{\partial R_{s, i}(p)}{\partial\left|g_{i}\right|}=2 p\left|g_{i}\right| \operatorname{MMSE}\left(\left|g_{i}\right|^{2} p\right) \leq 2 p\left|g_{i}\right|
\end{gathered}
$$

where $R_{s, i}(p)$ is defined in the expression of (7).

According to equation (22) and the Lagrange's mean value theorem [17, we have

$$
\left|R_{s, i}(p)-R_{s, i}\left(p^{\prime}\right)\right| \leq\left|h_{i}\right|^{2}|| p-p^{\prime} \|_{\infty} .
$$

On the other hand, combining (23), (24), (21), (18) and (19), using chain rule and the Lagrange's mean value theorem [17, we have

$$
\left|R_{s, i}(p)-R_{s, j}(p)\right| \leq\left(2 p\left|h_{i}\right| L_{h}+2 p\left|g_{i}\right| L_{g}\right) \frac{|i-j|}{N} .
$$

As $\left|h_{i}\right|,\left|g_{i}\right|$ and $p$ are bounded, according to (25) and (26), there must exist an $L$ such that

$$
\left|R_{s, i}(p)-R_{s, j}\left(p^{\prime}\right)\right| \leq L\left(\frac{|i-j|}{N}+\left\|p-p^{\prime}\right\|_{\infty}\right)
$$

i.e., the secrecy rate $R_{s, i}(p)$ is Lipschitz continuous. Hence according to Theorem 2 of [12], the duality gap between $D^{*}$ and $R^{*}$ vanishes asymptotically in the order of $1 / \sqrt{N}$, which is expressed as

$$
0 \leq D^{*}-R^{*} \leq O\left(\frac{1}{\sqrt{N}}\right),
$$

and Proposition 2 holds.

The procedures to solve (16) and (17) are provided in the following.

For each fixed $u$, problem (16) can be decoupled into $N$ independent subcarrier problems

$$
\begin{aligned}
g(u) & =\max _{p_{i} \geq 0} L\left(p_{i}, u\right), \\
& =\sum_{i=1}^{N} \max _{p_{i} \geq 0}\left[\left[I\left(s_{i} ; y_{i}\right)-I\left(s_{i} ; z_{i}\right)\right]^{+}-u p_{i}\right]+u P .
\end{aligned}
$$


Table 1.

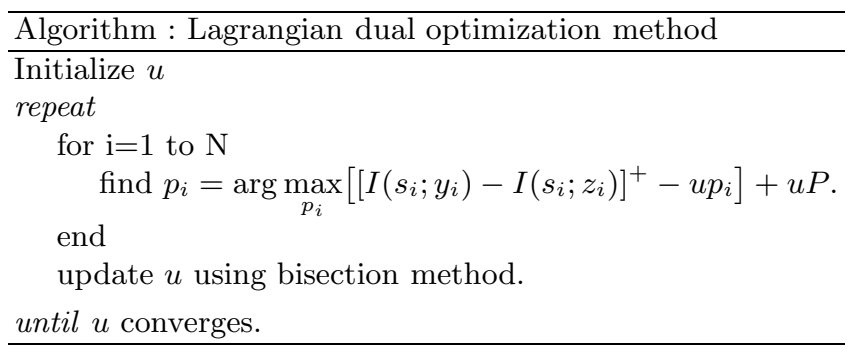

While the sub-carrier problem in (29) is still non-convex, it has only one variable $p_{i}$ and can be solved by simple one dimension line search. As the dual function $g(u)$ is convex in $u$, its subgradient $g^{\prime}(u)=P-\frac{1}{N} \sum_{i=1}^{N} p_{i}^{*}$, where $p_{i}^{*}$ is optimal solution for problem (16) with fixed $u$, is an increasing function in $u$. Hence bisection method can be used to solve dual problem (17), so that either $u=0$, $P \geq \frac{1}{N} \sum_{i=1}^{N} p_{i}^{*}$ or $u>0, P=\frac{1}{N} \sum_{i=1}^{N} p_{i}^{*}$ is satisfied. Table 1 summarizes the algorithm.

The complexity of this algorithm is $N \frac{1}{e_{p}} \log _{2}\left(\frac{1}{e_{d}}\right)$, where $e_{p}$ is the accuracy of one dimension exhausitive search to solve (16) and $e_{d}$ is the accuracy of the bisection search to solve (17). Since its complexity is linear with respect to the number of sub-carriers $N$, it is quite convenient for practical large values of $N$, such as $64 \sim 4096$. We note that the complexity of solve (6) exhaustively is $\frac{1}{e_{p}^{N}}$, which is exponential in $N$ and thus unrealistic.

\section{Numerical Results}

In this section, we provide some simulation results to illustrate the performance of our proposed power allocation algorithm and show how different channel input distributions affect the secrecy rate and power allocation results.

We first consider an OFDM-based secure system with $N=128$ sub-carriers. The secrecy rate versus total power constraint under different power allocation strategies and channel input distributions are illustrated in Fig. 4. Two reference strategies are considered to compare with our strategy: the optimal strategy under Gaussian inputs, i.e., (9), which is denoted by "PA of (9)" in Fig. 44 the equal power allocation strategy, which equally allocates total power among the sub-carriers that satisfy $\left|h_{i}\right|^{2}>\left|g_{i}\right|^{2}$ and is denoted by "equal PA".

Seen from Fig. (4 higher secrecy rate can be achieved for QPSK and 16QAM by our proposed optimal power allocation strategy, especially when the power constraint $P$ is quite large. However, equal power allocation and power allocation of (9) can be quite bad under finite discrete constellations, and the secrecy rate even drops to zero for large value of $P$. Actually, when $P$ is large, secrecy rate under Gaussian inputs can be approximated by 


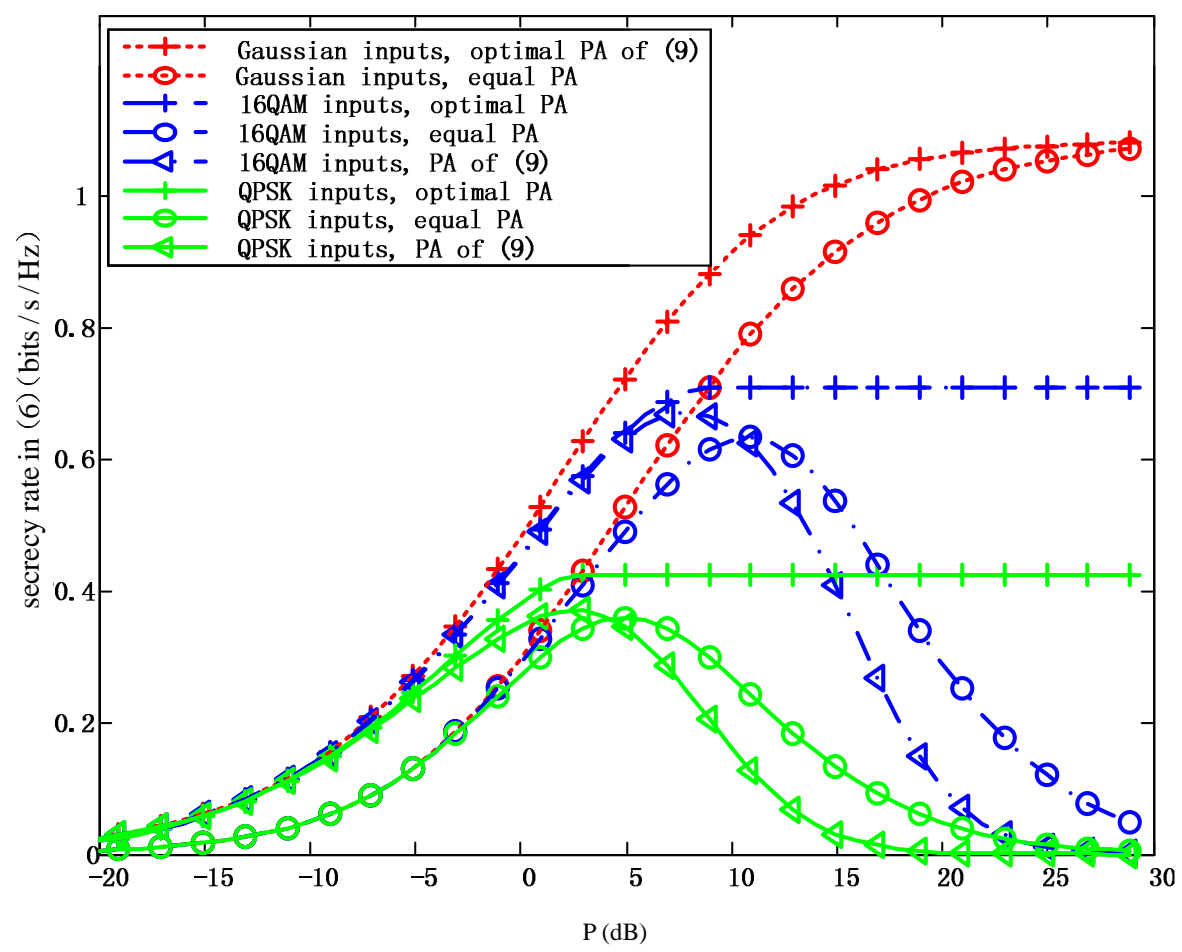

Fig. 4. The secrecy rate versus total power $P$.

$$
\begin{aligned}
R_{s}^{G}(\mathbf{p}) & =\frac{1}{N} \sum_{i=1}^{N}\left[\log _{2}\left(1+\left|h_{i}\right|^{2} p_{i}\right)-\log _{2}\left(1+\left|g_{i}\right|^{2} p_{i}\right)\right]^{+} \\
& \approx \frac{1}{N} \sum_{i=1}^{N}\left[\log _{2}\left(\frac{\left|h_{i}\right|^{2}}{\left|g_{i}\right|^{2}}\right)\right]^{+}
\end{aligned}
$$

which is independent with power allocation $p_{i}$. So equal power allocation works asymptotically optimal under Gaussian inputs when $P$ is large, as shown in Fig. 4.

The power allocation solution of the proposed algorithm is shown in Fig. 5 and Fig. 6, respectively, under QPSK and Gaussian inputs with $N=4$. When the power constraint $P$ is small, most transmit power is allocated to the stronger sub-channels, the channels with larger $\left|h_{i}\right|^{2}-\left|g_{i}\right|^{2}$ (Channel 2 and Channel 4 in our simulation example). However, as $P$ grows, the transmit power allocated to the weak sub-channels grows. Under QPSK input signals, the transmit power allocated to every sub-channel should stop increasing when $P$ is very large. But the transmit power under Guassian input signals keeps increasing.

\section{Conclusion}

In this paper, we have obtained the optimal power allocation for OFDM-based wire-tap channels with arbitrarily distributed inputs. While the secrecy rate 


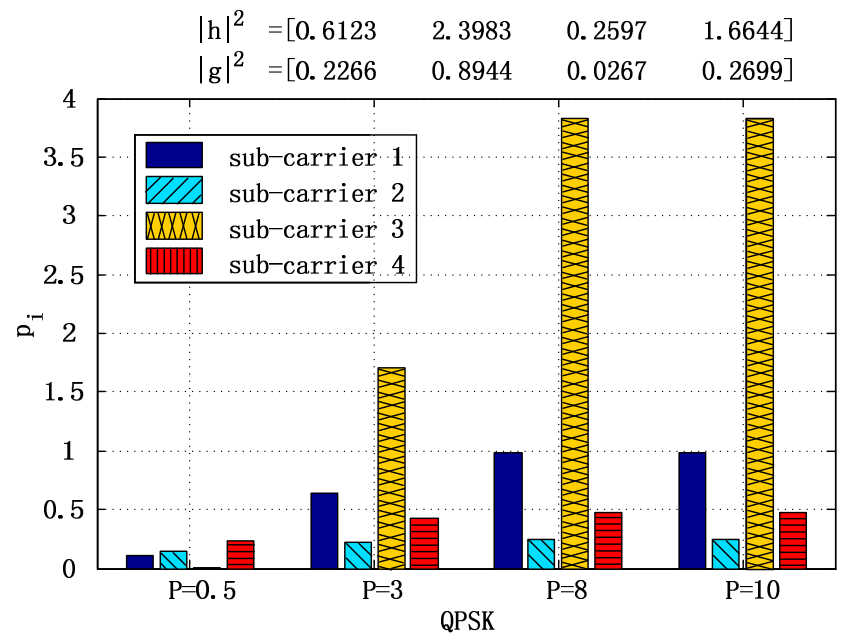

Fig. 5. Power allocation results versus $P$ under QPSK inputs.

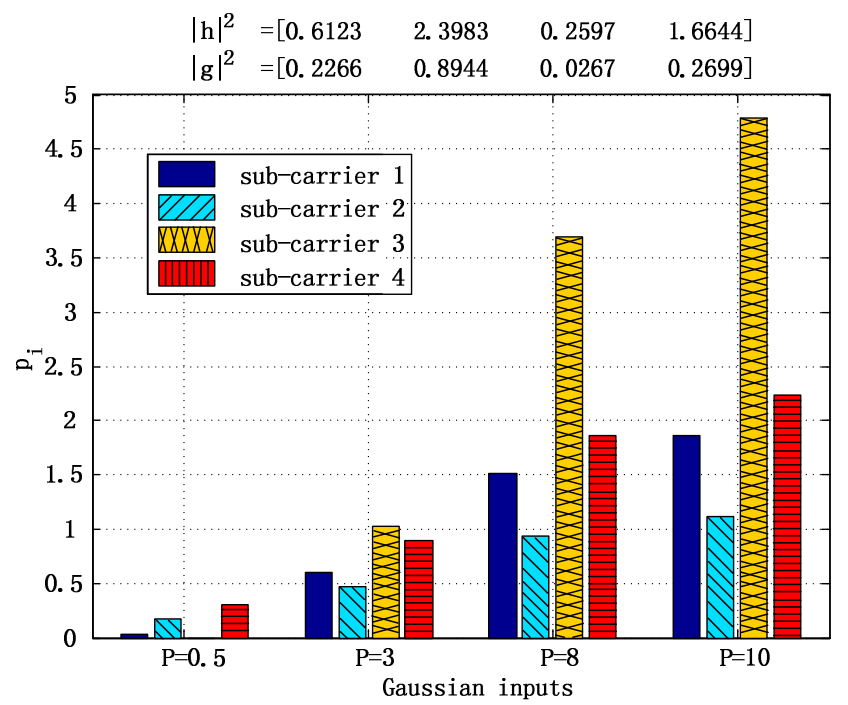

Fig. 6. Power allocation results versus $P$ under Gaussian inputs.

achieved by Gaussian distributed channel inputs is concave with respect to the transmit power, we have found and rigorously proved that the secrecy rate is non-concave under any practical finite discrete constellations. A power allocation algorithm has been proposed, which is asymptotic optimal as the number of sub-carrier increases. Our numerical results show that more transmit power may results in a huge loss in secrecy rate, which is rarely seen in previous power allocation studies. This indicates that optimal power allocation is quite essential in practical studies of physical layer security. 
Acknowledge The authors would like to thank Dr. Tsung-Hui Chang and Prof. Shidong Zhou for their valuable suggestions in this paper.

This work is supported by National S\&T Major Project (2009ZX03002-002), National Basic Research Program of China (2007CB310608), National S\&T Pillar Program (2008BAH30B09), National Natural Science Foundation of China (60832008) and PCSIRT, Tsinghua Research Funding-No.2010THZ02-3. This work is also sponsored by Datang Mobile Communications Equipment Co., Ltd.

\section{References}

1. Y. Liang, H. V. Poor, and S. Shamai (Shitz), "Information theoretic security," Found. Trends Commun. Inf. Theory, vol. 5, pp. 355-580, 2008.

2. A. Wyner, "The wire-tap channel," Bell Syst. Tech. J., vol. 54, no. 8, pp. 1355-1387, Jan. 1975.

3. I. Csiszar and J. Korner, "Broadcast channels with confidential messages," IEEE Trans. Inf. Theory, vol. 24, no. 3, pp. 339-348, May 1978.

4. S. L. Y. Cheong and M. Hellman, "The Gaussian wire-tap channel," IEEE Trans. Inf. Theory, vol. 24, no. 4, pp. 451-456, July 1978.

5. F. Oggier, and B. Hassibi, "The secrecy capacity of the MIMO wiretap channel," in Proc. 45th Annu. Allerton Conf. Communication, Control and Computing, Monticello, IL, Sept. 2007, pp. 848-855.

6. T. Liu, and S. Shammai(Shitz), "A note on the secrecy capacity of the multi-antenna wiretap channel," IEEE Trans. Inf. Theory, vol. 55, no. 6, pp. 2547-2553, Jun. 2009.

7. E. Ekrem, and S. Ulukus, "Gaussian MIMO multi-receiver wiretap channel," Global Telecommunications Conf., Honolulu, HI, Nov. 2009.

8. Z. Li, R. Yates, and W. Trappe, "Secrecy capacity of independent parallel channels," in Proc. 44th Annu. Allerton Conf., Allerton House, Illinois, pp. 841-848, Jul. 2006.

9. Y. Liang, H. V. Poor and S. Shamai(Shitz), "Secure communication over fading channels," IEEE Trans. Inf. Theory., vol. 54, no. 6, pp. 2470-2492, Jun. 2008.

10. E. Jorswieck and A. Wolf, "Resource allocation for the wire-tap multi-carrier broadcast channel," in Proc. International Workshop Multiple Access Communications Conf., St. Petersburg, Russia, June 2008.

11. F. Renna, N. Laurenti and H. V. Poor, "Physical layer security for OFDM systems," European Wireless Conf.,Vienna, Austria, Apr. 2011.

12. Z. Luo, and S. Zhang, "Duality Gap Estimation and Polynomial Time Approximation for Optimal Spectrum Management," IEEE Trans. Signal Process., vol. 57, no. 7, pp. 2675-2689, July 2009.

13. Z. Luo and S. Zhang, "Dynamic spectrum management: Complexity and duality," IEEE J. Sel.Top. Signal Process., Special Issue on Signal Process. Netw. Dyn. Spectrum Access, vol. 2, no. 1, pp. 57-73, Feb. 2008.

14. W. Yu and R. Lui, "Dual methods for nonconvex spectrum optimization of multicarrier systems," IEEE Trans. Commun., vol. 54, pp. 1310-1322, July. 2006.

15. D. Guo, S. Shamai (Shitz), and S. Verdu, "Mutual information and minimum mean-square error in Gaussian channels," IEEE Trans. Inf. Theory, vol. 51, no. 4, pp. 1261-1283, Apr. 2005.

16. D. Guo, Y. Wu, S. Shamai, and S. Verdu, "Estimation in Gaussian noise: Properties of the minimum mean-square error," IEEE Trans. Inf. Theory, vol. 57, no. 4, pp. 2371-2385, April 2011. 
17. H. Jeffreys and B. S. Jeffreys, Methods of Mathematical Physics, Cambridge, England: Cambridge University Press, 3rd ed., 1988.

18. L. Boyd, S. Vandenberghe, Convex Optimization, Cambridge, England: Cambridge University Press, 2004. 